\title{
Surfer's myelopathy: case report and review
}

\author{
Amna Karabegovic, MD, MSc ${ }^{{ }^{\dagger}}$; Shirley Strachan-Jackman, RNP*; David Carr, MD* ${ }^{\dagger}$
}

\section{ABSTRACT}

Nontraumatic spinal cord injury from surfing is a new entity first described in 2004 and likely of ischemic etiology. We report the case of a 25-year-old man who presented to the emergency department with a 2-week history of lower extremity weakness after surfing in Indonesia. The patient reported developing low back pain, lower extremity weakness, sensory changes, and urinary retention shortly after his first surfing lesson. The patient was subsequently diagnosed with surfer's myelopathy. The purpose of this report is to review the clinical presentation, etiology, risk factors, and management of this increasingly described entity.

\section{RÉSUMÉ}

Les blessures à la moelle épinière non traumatiques causées par le surf constituent une nouvelle entité, décrite pour la première fois en 2004 et d'étiologie ischémique probable. Nous faisons état du cas d'un homme de 25 ans qui s'est présenté au service des urgences avec un historique de faiblesse aux extrémités depuis 2 semaines, après avoir fait du surf en Indonésie. Le patient a rapporté avoir développé une douleur au bas du dos, une faiblesse des extrémités inférieures, des modifications sensorielles et de la rétention urinaire peu de temps après sa première leçon de surf. On a par la suite diagnostiqué au patient une myélopathie du surfeur. Le but de ce rapport est de faire la revue du tableau clinique, de l'étiologie, des facteurs de risque et de la gestion de cette entité de plus en plus souvent mentionnée.

Keywords: myelopathy, paraparesis, spinal cord injury, surfer, surfing

It is estimated that over 18 million individuals surf worldwide. ${ }^{1}$ As surfing grows in popularity, surfing injuries are undoubtedly becoming more common. The increasing popularity of this sport is likely attributable, at least in part, to the commercialization of surfing apparel and the freedom and exhilaration of the stereotypical surfing lifestyle. Craniospinal injuries associated with surfing have been previously reported. These injuries are usually traumatic secondary to head impact with the sea floor or due to a direct blow by a surfboard. ${ }^{1}$ Surfer's myelopathy is a nontraumatic spinal cord injury that affects novice surfers. To date, only 10 cases of this condition have been reported in the medical literature, and surfer's myelopathy is believed to be an underdiagnosed and thus underreported condition. ${ }^{2,3} \mathrm{We}$ describe a case of a beginner surfer with lower thoracic spinal cord injury that developed insidiously after his first surfing lesson and then review the literature on this condition.

\section{CASE REPORT}

A 25-year-old previously healthy man took a 1-hour surfing lesson in Bali, Indonesia. He reported no untoward traumatic events while surfing. Approximately an hour later, he began to experience low back pain radiating down to his lower limbs, paresthesias, and bilateral lower extremity weakness. In the subsequent hours, he developed urinary retention, increasing abdominal pain, and difficulty ambulating. A hotel doctor saw him, and he was catheterized for $2 \mathrm{~L}$ of urine. As he remained in urinary retention, he was assessed the next day at an international clinic in Bali. A Foley catheter was inserted and drained of $1 \mathrm{~L}$ of urine. According to the notes from the clinic, his vital signs were stable, with unremarkable cardiovascular and respiratory examinations. He had suprapubic tenderness, and a neurologic examination revealed bilateral lower extremity weakness, with power rated as

From the *Department of Emergency Medicine, Toronto Western Hospital, University Health Network, Toronto, ON; †Department of Family and Community Medicine, University of Toronto, Toronto, ON.

Correspondence to: Dr. David Carr, Emergency Department, Toronto Western Hospital, University Health Network, 399 Bathurst Street, Toronto, ON M5T 2S8; e-mail: david.carr@uhn.on.ca.

Submitted August 13, 2010; Revised December 5, 2010, and December 20, 2010; Accepted December $24,2010$.

This article has been peer reviewed.

(c) Canadian Association of Emergency Physicians 
$4 / 5$. He was diagnosed with a urinary tract infection and muscle weakness secondary to overuse. No diagnostic imaging was performed on his initial presentation, and he was started on a 7-day course of ciprofloxacin.

He presented to a hospital in Jakarta 7 days later, reporting persisting weakness, sensory deficits in the legs, urinary retention, and fecal incontinence. There he was assessed by both the urology and neurology services and was subsequently admitted to hospital. His examination revealed bilateral spasticity of his lower extremities with hyperreflexia and clonus. In addition, his sensory examination showed hypoesthesia of the lower extremities below the level of the thoracic region in a nondermatomal distribution. At the time, only a magnetic resonance image (MRI) of the neck was obtained, which revealed cervical spine degenerative changes. He was diagnosed with an upper motor neuron lesion secondary to "spinal cord concussion" and treated with a 2-day course of steroids. Within 48 hours, the patient was able to ambulate and was discharged from the hospital. He subsequently flew back to Canada.

Three days later, he presented to our emergency department in Toronto with complaints of residual paraparesis, hypoesthesias, and urinary hesitancy. Physical examination revealed an antalgic gait. Deep tendon reflexes were $2+$ in the upper limbs and $4+$ in the lower limbs and symmetric. The plantar responses were both upgoing. He had decreased cold and vibration sensation in his feet and urinary hesitancy with a postvoid residual volume of $113 \mathrm{~mL}$. His rectal tone was normal. A contrast-enhanced MRI of the spine was unremarkable. He was seen by the neurology service and was diagnosed with "transient myelopathy not yet diagnosed." Their opinion was that he had residual evidence of spinal cord dysfunction despite his negative MRI. The conclusion was that the patient was recovering from a myelopathy, likely surfer's myelopathy.

\section{DISCUSSION}

Surfer's myelopathy is an acute nontraumatic myelopathy typically affecting the thoracolumbar spine. The incidence is unknown, but first-time surfers are believed to be at significant risk. To date, 10 cases have been reported in the literature of nontraumatic spinal cord injury associated with surfing. ${ }^{2,3}$ In the first report, Thompson and colleagues described nine patients with surfer's myelopathy between June 1998 and January 2003. ${ }^{2}$ The average age was 25 years, and the common features included inexperience (first-time surfers), a thin, underdeveloped body habitus, dehydration, and long-distance travel to the surfing destination. All of the reported patients had abnormal thoracolumbar spinal cord findings on MRI but no evidence of fracture, spondylolysis, spondylolisthesis, spinal stenosis, or acute disk herniation. Of the nine patients described by Thompson and colleagues, four had complete recovery and four had persistent lower extremity weakness with normal sensory function (three of whom had persistent urinary retention). One patient remained paraplegic. Aviles-Hernandez and colleagues reported a case of a 37-year-old local Hawaiian resident who developed features consistent with surfer's myelopathy after taking a 2-hour surfing lesson. ${ }^{3}$ Hence, the common trait in all 10 cases seems to be inexperience.

Thompson and colleagues proposed an ischemic event as an underlying etiology for surfer's myelopathy. ${ }^{2}$ As nonexperienced surfers spend most of their lesson paddling in a hyperextended position on the board, this is hypothesized to compromise thoracolumbar blood flow, leading to ischemia. The spinal cord receives its circulatory supply from the anterior spinal artery and two posterior spinal arteries, the latter of which supplies the cervical level. Radicular arteries provide circulation to the lower spine, of which the artery of Adamkiewicz provides the major blood supply to the inferior two-thirds of the spinal cord. Hence, the primary watershed area of the spinal cord is the midthoracic region. ${ }^{4}$ Given that all of the patients in the Thompson and colleagues report developed MRI changes in the midthoracic spine, the authors postulated that a hyperextended posture during surfing leads to infarction at the level of the watershed zone. Suggested mechanisms include avulsion of perforating vessels, vasospasm of the artery of Adamkiewicz, or transient ischemia in areas of borderline perfusion as a result of tension on the spinal cord with hyperextension. ${ }^{2}$ Spinal cord ischemia secondary to spine positioning has been reported in operating room patients after being placed in extreme lordosis for a prolonged period of time,, 5 and MRI studies of such patients reveal abnormal signal changes suggestive of thoraciclevel ischemia.

Although less likely than arterial insufficiency, venous infarction is another possible etiology of 


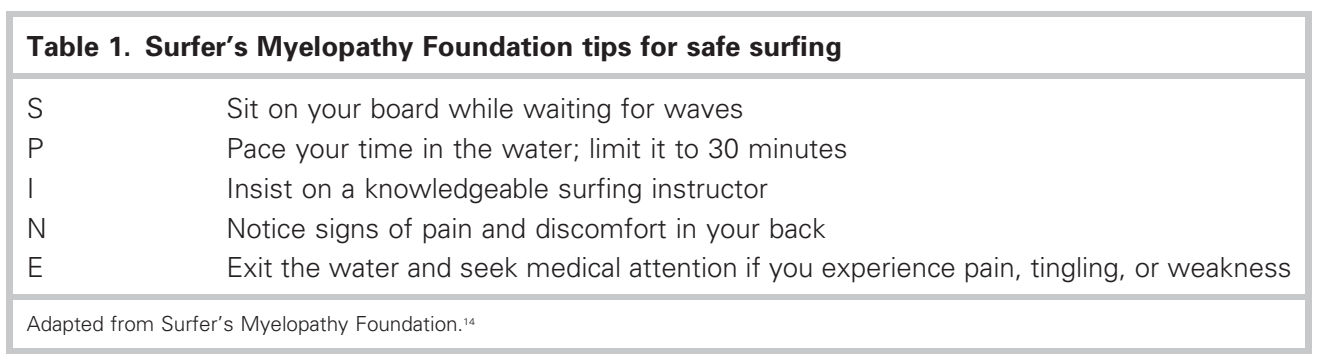

surfer's myelopathy. Aviles-Hernandez and colleagues described possible inferior vena cava obstruction and fibrocartilaginous embolism as a possible mechanism of injury. ${ }^{3}$ It is proposed that compression of the inferior vena cava by the liver while lying on the surfboard and prolonged Valsalva maneuver during paddling may lead to increased retrograde pressure in the epidural venous plexuses, leading to infarction. ${ }^{3,7}$

There are other important entities to consider in the differential diagnosis of a patient presenting with clinical findings similar to the case we describe. These include transverse myelitis, viral myelitis, Guillan-Barré syndrome, cauda equina syndrome, and spinal cord syndromes such as Brown-Séquard syndrome. Through a careful history and physical examination, along with laboratory and appropriate imaging, the exclusion of these uncommon entities can be facilitated.

Thompson and colleagues suggested that the initial assessment and management of a patient suspected of surfer's myelopathy should include hemodynamic management, an urgent MRI, and a urologic evaluation with urodynamic studies. ${ }^{2}$ More specifically, several studies have suggested that MRI with diffusionweighted imaging (DWI) is the single most important imaging study in the initial evaluation of suspected spinal cord infarction. ${ }^{3,8-10}$ The process of restricted water diffusion seen after the ischemic insult occurs over the course of minutes; hence, DWI has a high sensitivity and will be positive within the first few hours, whereas the initial changes in a $T_{2}$-weighted signal can take anywhere from 8 to 24 hours to develop. ${ }^{3}$

Currently, there is no standardized treatment protocol for ischemic spinal cord injuries. The use of high-dose steroids in patients with blunt trauma to the spine was suggested to improve neurologic function in a controversial 1990 National Acute Spinal Cord Injury Study. ${ }^{11}$ Steroid treatment of nontraumatic ischemic injuries of the spine has not been studied.
In the review by Cheshire and colleagues, $25 \%$ of cases had good spontaneous recovery, $50 \%$ had some degree of recovery, and $25 \%$ had unchanged symptoms. ${ }^{12}$

Like the nine cases in Thompson and colleagues' report, ${ }^{2}$ our patient possessed all the risk factors that could predispose him to surfer's myelopathy. In the case we describe, no MRI study was done in Bali, and the MRI report from Jakarta was limited to the cervical spine. By the time we saw the patient, he had improved clinically and there were no abnormalities noted on the MRI, a finding that could be consistent with the resolution of signal change. Although DWI MRI is an important diagnostic tool for examining patients with suspected spinal cord ischemia, many such patients have normal follow-up MRIs. ${ }^{13}$

To spread awareness of and to promote safety and injury prevention in the surfing community, the Surfer's Myelopathy Foundation has issued tips for safe surfing under the mnemonic "SPINE," as outlined in Table $1 .^{14}$

\section{CONCLUSION}

The growing popularity of surfing will likely result in increased incidence of both traumatic and nontraumatic spinal cord injuries from this sport. Surfer's myelopathy is a nontraumatic spinal cord injury, likely caused by ischemia secondary to spine hyperextension. Risk factors include first-time surfers, dehydration, long-distance travel, and weak baseline muscle tone. Initial interventions should include hemodynamic and urologic management and an urgent MRI. Most patients fully recover, but complete paraplegia has been described.

Competing interests: None declared.

\section{REFERENCES}

1. Nathanson A, Haynes P, Galanis D. Surfing injuries. Am 7 Emerg Med 2002;20:155-60, doi:10.1053/ajem.2002.32650. 
2. Thompson TP, Pearce J, Chang G, et al. Surfer's myelopathy. Spine (Phila Pa 1976) 2004;29:E353-6, doi:10.1097/ 01.BRS.0000134689.84162.E7.

3. Aviles-Hernandez I, Garcia-Zozaya I, DeVillasante JM. Nontraumatic myelopathy associated with surfing. I Spinal Cord Med 2007;30:288-93.

4. Ropper AH, Adams RD, Victor M, et al, Adams and Victor's principles of neurology. 9th ed. New York: McGraw-Hill Medical; 2009.

5. Roberts DR, Roe J, Baudouin C. Hyperlordosis as a possible factor in the development of spinal cord infarction. $\mathrm{Br} \mathrm{F}$ Anaesth 2003;90:797-800, doi:10.1093/bja/aeg136.

6. Beloeil H, Albaladejo P, Hoen S, et al. Bilateral lower limb hypoesthesia after radical prostatectomy in the hyperlordotic position under general anesthesia. Can 7 Anaesth 2003;50: 653-6, doi:10.1007/BF03018706.

7. Naiman JL, Donohue WL, Prichard JS. Fatal nucleus pulposus embolism of spinal cord after trauma. Neurology 1961;11:83-7.

8. Kuker W, Weller M, Klose U, et al. Diffusion-weighted MRI of spinal cord infarction-high resolution imaging and time course of diffusion abnormality. 7 Neurol 2004;251:81824, doi:10.1007/s00415-004-0434-z.
9. Shinoyama M, Takahashi T, Shimizu H, et al. Spinal cord infarction demonstrated by diffusion-weighted magnetic resonance imaging. 7 Clin Neurosci 2005;12:466-8, doi:10. 1016/j.jocn.2004.01.010.

10. Thurnher MM, Bammer R. Diffusion-weighted MR imaging (DWI) in spinal cord ischemia. Neuroradiology 2006;48: 795-801, doi:10.1007/s00234-006-0130-z.

11. Bracken MB, Shepard MJ, Collins WF, et al. A randomized, controlled trial of methylprednisolone or naloxone in the treatment of acute spinal-cord injury. Results of the Second National Acute Spinal Cord Injury Study. $N$ Engl 7 Med 1990;322:1405-11, doi:10.1056/NEJM199005173222001.

12. Cheshire WP, Santos CC, Massey EW, et al. Spinal cord infarction: etiology and outcome. Neurology 1996;47:32130 .

13. Masson C, Pruvo JP, Meder JF, et al. Spinal cord infarction: clinical and magnetic resonance imaging findings and short term outcome. $\mathcal{F}$ Neurol Neurosurg Psychiatry 2004;75:1431-5, doi:10.1136/jnnp.2003.031724.

14. Surfer's Myelopathy Foundation. Tips for first time surfers. 2008. Available at: http://www.smawareness.org/tips.html (accessed July 19, 2010). 